\title{
SONIFIGRAPHER. SONIFIED LIGHT CURVE SYNTHESIZER
}

\author{
Adrián García Riber \\ Image and Sound Art, \\ Francesc Martí i Mora 1-B 22-3, \\ Palma de Mallorca, 07011, Spain \\ adrian@imageandsoundart.com
}

\begin{abstract}
In an attempt to contribute to the constant feedback existing between science and music, this work describes the design strategies used in the development of the virtual synthesizer prototype called Sonifigrapher. Trying to achieve new ways of creating experimental music through the exploration of exoplanet data sonifications, this software provides an easy-touse graph-to-sound quadraphonic converter, designed for the sonification of the light curves from NASA's publiclyavailable exoplanet archive. Based on some features of the first analog tape recorder samplers, the prototype allows end-users to load a light curve from the archive and create controlled audio spectra making use of additive synthesis sonification. It is expected to be useful in creative, educational and informational contexts as part of an experimental and interdisciplinary development project for sonification tools, oriented to both non-specialized and specialized audiences.
\end{abstract}

\section{INTRODUCTION}

According to Vickers' [1] distinction between auditory and sonified graphs, Sonifigrapher can be defined as a virtual synthesizer that provides non-MIDI sonified graphs through the exploration and mapping of the RGB values of user-loaded PNG files. Initially inspired by the Chamberlin and the Mellotron concept, where each key of a keyboard reproduces an analog tape pre-recorded sound, Sonifigrapher synthesizer works as an image sonification sampler that allows single playing and looping. The prototype has been initially developed to create sonifications from the publicly-available graphic astronomical information published at Mikulski Archive for Space Telescopes (MAST) [2,3], the simulated light curves of the Planet Hunters project from the Transiting Exoplanet Survey Satellite (TESS) [4,5], and the curves generated with the Lightkurve software package for Kepler \& TESS time series analysis in Python [6]. However, its design can be easily adapted to any kind of graphic representation

\section{REFERENCE WORKS}

In 1980, motivated by his interest in the direct synthesis of the time pressure curve [7], Iannis Xenakis started developing the Unité Polyagogique Informatique du CEMAMu (UPIC) which by the nineties, already represented a new paradigm in the use of experimental music devices for learning [8]. Created as a mouse-controlled, graphical-musical composing system [9], UPIC allowed musicians to give orders to the computer through drawings [10].
This inspiring concept underlies numerous projects focused on graphic to sound conversion and laid the foundations of the graphical open-source sequencer for digital art IanniX [11], [12]. Further from the artistic point of view and designed for the creation of multi-purpose auditory graphs, Sonification Sandbox by Walker \& Cothran [13], provides a multiplatform MIDI-based, graph-to-sound, userselectable sonification engine, based on previous projects MUSE and MUSEART. It was designed for a wide range of users, from novice to expert, and allows adding context to the sonified data using reference tracks. In a more specific approach, Bell3D audio-based Astronomy Education System [14] and xSonify astronomical data sonification software [15], represent two reference examples of sonification projects designed to make data-driven astronomy accessible to both visually impaired and sighted users. The first one, by Jaime Ferguson, allows users to learn about basic astronomy through surround sonifications of user-selected stars' parameters. The xSonify project from Diaz-Merced et al. [16], sonifies twodimensional data from text files, for large data sets in frequency dimensions, trying to find visually masked correlations and patterns.

\section{ABOUT LIGHT CURVES AND TRANSITS}

Light curves are graphic representations of the brightness flux variations along time, observed in celestial objects. When a planet passes in front of a star, it generates a partial eclipse called transit, producing a flux decrement in its light curve which can be measured in terms of time and depth.

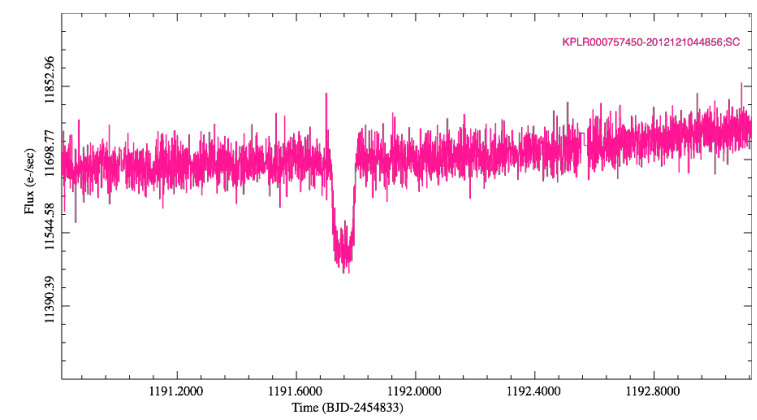

Figure 1: Enlarged view of KPLR000757450-2012121044856 light curve showing a planet transit [17]. PDCsap Bright Flux (Pre-search Data Conditioning Simple Aperture Photometry, electrons per second) [18] vs time expressed in BJD-2454833 (Barycentric Julian Date, 2454833.0 offset) [19].

In 1999, HD 209458 b, nicknamed 'Osiris', was the first exoplanet to be seen in transit around its star, opening new horizons in exoplanet characterization through the transit detection method [20]. Currently, the number of confirmed 
exoplanets is about four thousand and increasing every week (3972 confirmed exoplanets, 05/26/2019) [21].

As described by Seager \& Mallén-Ornelas [22], the planet's orbit can be characterized by analyzing the decrease of energy presented in the light curve. Measuring time between transits, the orbital period is also obtained to complete the equations.

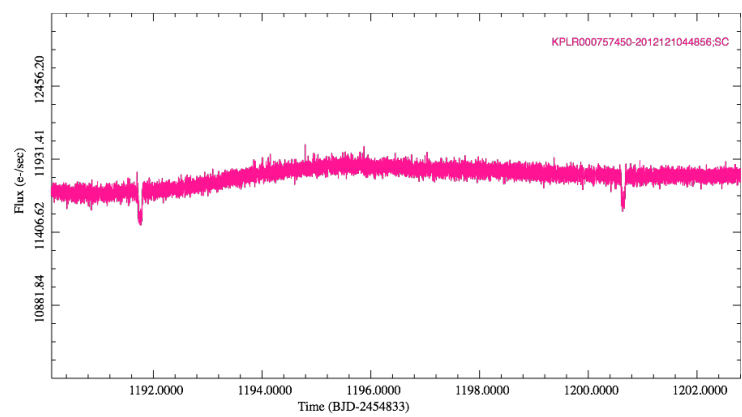

Figure 2: KPLR000757450-2012121044856 light curve showing two transits [17]. PDCsap Flux (electrons per second) [18] vs Time (BJD-2454833) [19].

In addition to transit photometry, the radial velocity of the host star is needed to determine planet's radius and mass. This method detects the oscillating Doppler shift variation in the radial velocity of the star due to the gravitation of an orbiting planet [22]. The planet's temperature and atmospheric properties can also be determined through transmission spectroscopy methods consisting of the observation of transit light curves at different wavelengths [23].

However, the detection and characterization of exoplanets is not free of challenges and many factors make it usual to generate false positives or loss of information and uncertainties. Worth mentioning that stellar systems such as Brown Dwarfs or Eclipsing Binaries can produce variations in the light curves similar to those generated by the orbiting planets. The stellar activity can also affect the observation of the exoplanet's atmosphere and inherent noise can affect the detection of the weakest planet's signals [24].

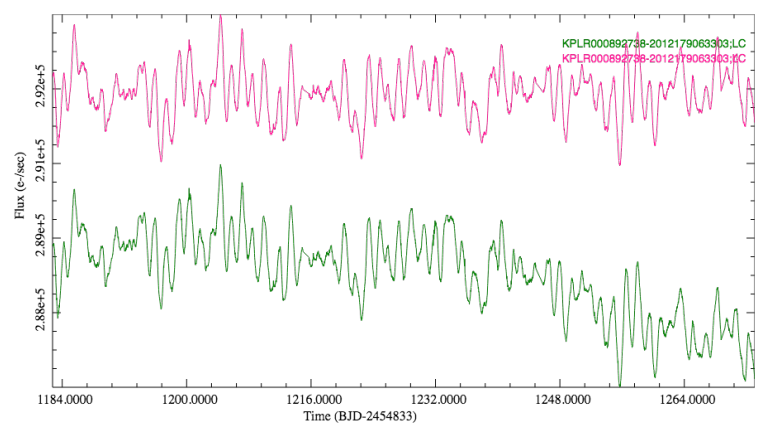

Figure 3: KPLR000892738-2012179063303. Possible Red Giant star [17]. PDCsap Flux (red) Sap Flux (green) [18] vs Time (BJD-2454833) [19].

\section{VIRTUAL INSTRUMENT DESIGN}

Sonifigrapher is a quadraphonic/stereo virtual instrument prototype designed for sonifying light curves. In the same way as wavetable synthesizers work, it makes use of the curves to generate filter-controlled audio spectra through additive synthesis which control variables have been mapped from the graphic representation input. Csound's [25] backwards and future compatibility compromise, together with the possibilities that its API for Python [26] and Cabbage's [27] VST plug-in exporting option provide, induced the workflow decision for the project.

Subscribing the words by Gerhard Steinke related to newly developed electronic music instruments expressing that "a great number of composers should be given the possibility of interpreting their ideas in various ways after having become familiar with the apparatus" (about his subharmonic synthesizer prototype, Subharchord, 1966) [28], Sonifigrapher can be downloaded for testing as a packed 'ZIP' file from:

https://archive.org/details/SonifigrapherMacOSX

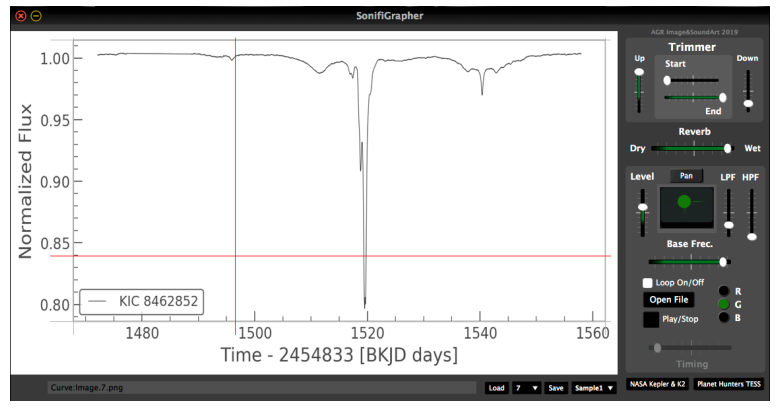

Figure 4: Sonifigrapher interface synthesizing the unusual light curve of Tabby's star, extracted from Lightkurve Python's package website [6].

A first stage of intensive testing with Marilungo's examples of CSound's image processing opcodes [29], [30] was crucial to motivate the development of Sonifigrapher prototype. These reference approaches made it possible to summarize the benefits and drawbacks of sonified graphs as well as to experiment with the aesthetics and accuracy aspects of the final design. McCurdy's Cabbage and CSound examples [31] were also consulted for technical resolution strategies in the final implementation.

The design of the prototype relies on the main pillars of sonification processes highlighted by Scaletti [32], Hermann [33], [34], De Campo [35], Vogt [36] or Kramer et al. [37], assuring:

- Original information communication.

- Adaptation to the language and needs of the research field.

- Systematic transformation of the input data.

- End-user control and/or interaction.

- Reproducibility.

- Possibility of validation and repetition with different input data sets.

- Integrability.

The final synthesizer has been developed to meet the following goals:

- To explore the multimodal display possibilities of CSound and Cabbage workflow.

- To provide a sonification tool for testing auditory transit detection.

- To demonstrate the added value of multimodal synergies in graphic datasets.

- To generate a cross-domain, interference-preserving mapping. 
- To create a multimodal tool for approaching and/or communicating the information contained in light curves databases from different perspectives within creative, informational and/or educational contexts.

- To implement an extremely intuitive UI with almost no learning curve.

- To allow real-time operation and live performance.

- To integrate the virtual instrument in Digital Audio Workstations.

\section{IMPLEMENTATION ALGORITHM}

In order to generate a sound representation allowing accurate perception of graphic changes and according to the aesthetics of Experimental, Electronic and Electroacoustic music, Sonifigrapher uses additive synthesis with a non-quantified frequency scale generated from a user-defined base frequency. This approach makes it possible to create tonal sweeps and microtonal sounds or chords and improves accuracy in light curves' pitch tracking. As the final sonified spectrum relies on a user-defined base frequency, it is possible to adapt the graphic changes in the curves to different frequency ranges for a better perception of the sonification, or fine-tuning in creative applications. To maintain coherence with the transit detection method, the lowest flux values in the curves correspond to the highest frequencies in the sonification. In this way, when a transit is produced, a high frequency sine is reproduced facilitating its detection.

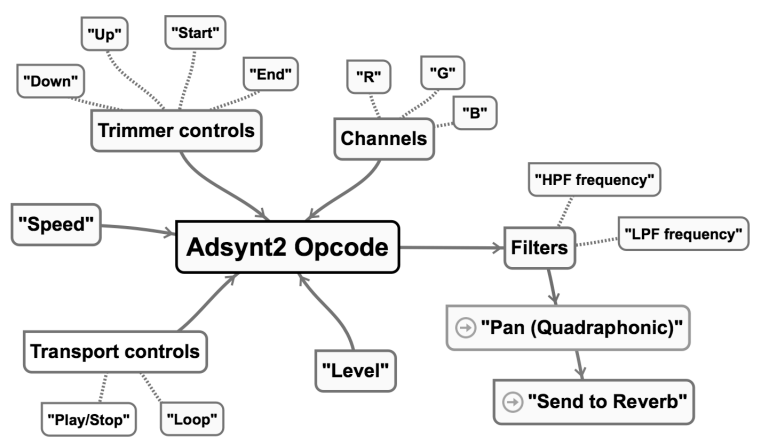

Figure 5: Sonifigrapher design map showing control variables and signal flow.

The core of the prototype is therefore CSound's adsynt2 opcode [29], which performs additive synthesis with an arbitrary number of partials, not necessarily harmonics. This opcode also provides interpolation to lightly soften the most pronounced graphic transitions. Figure 5 describes the design implementation map with all the variables used to control the sonification process. The R, G and B values of the loaded image are extracted using CSound's image processing opcodes [29] to work as input arguments for adsynt2. Its monophonic output is low- and high-pass filtered and sent in parallel to a quadraphonic matrix and reverberation processor to be used in creative applications. End users can select the sonified R, B or G channel input to reduce noise and focus attention.

The synthesizer also provides trimmer controls to adjust the 'start' and 'end' points of reproduction as well as the 'up' and 'down' graphic limits to avoid the sonification of nonrelevant information printed in the sampled image. The speed control allows both detailed analysis and fast monitoring, if the loop playback is not enabled. The loop reproduction works on an eight seconds Mellotron-based time scale and disables timing control. All changes made to the 'trimmer', 'speed' and 'loop' controls are applied once the current reproduction is completed. High- and low-pass filters frequencies are controlled before the signal is sent to the reverberation processor to improve sound quality. A user controlled " $x-y$ " matrix is also provided for sound allocation in a quadraphonic reproduction system. Default auto-panning configuration follows the graphic timeline bar with stereo compatibility. The 'level' fader acts over both the 'dry' and 'wet' signals by minimizing the number of controls required.

Going further on the sonification process through adsynt 2 opcode control, next figure describes the input and output arguments being used by the algorithm (referred to CSound variables). The complete source code is available in the '.csd' file included in the downloadable version of Sonifigrapher.

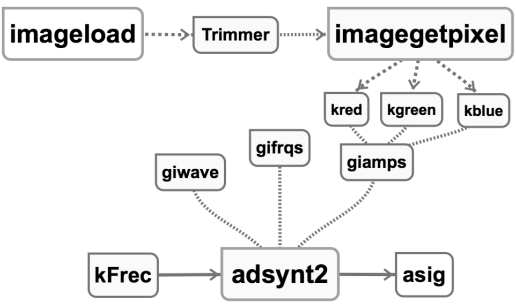

Figure 6: Design map showing CSound's adsynt2, imageload and imagegetpixel opcodes with input and output arguments. (Based on Marilungo's work).

Once the image is loaded, four global ' $i$ ' variables (gilmageIni, gilmageEnd, giVLimUp and giVLimDw), hold the trimmer options before the extraction of every pixel's bright amplitude in the $\mathrm{x}-\mathrm{y}$ exploration process. These amplitudes are stored in three ' $k$ ' variables (kred, kgreen and kblue), used to control the amplitude ratios of the generated sine waves through a global 'i' variable (giamps).

If the prototype is loaded with a white background plot, these values are inverted using a threshold detection conditional statement to reduce background noise related to high bright pixel values. End users can select the RGB channel to be sonified (gkR, gkG and gkB in the source code), and adapt the base frequency ( $\mathrm{kFrec}$ ) of the synthesized spectrum to their needs. The total number of sinusoids and the frequency ratios are introduced in the opcode through two global ' $\mathrm{i}$ ' variables (giwave and gifrqs).

The synthesized signal (asig), is passed through two cascade high- and low-pass filters, generating the mono filtered audio output (aFilt). This signal is routed to four audio output channels using two parallel pan 2 opcodes that allow front and rear panning (gaLf, gaRf, gaLr and gaRr). The reverberation effect has been implemented in an independent instrument for best audio quality and makes use of two independent processors fed by the same stereo front audio signals (gaSenL and gaSendR), enhancing the surround environment. The master level of the four output channels is controlled with a single global ' $\mathrm{k}$ ' variable (gkAmp).

\section{ACCESING THE DATABASE}

To simplify the access to the light curve database, the prototype includes a 'NASA Kepler\&K2' button which links to NASA's description of 'Two ways to get Kepler Light Curves' [2]. The 
following instructions allow the reproduction of the light curve showed in Figure 1.

- Go to: http://archive.stsci.edu/kepler/data_search/search.php

- Press the 'Search' button to access the complete catalog.

- Mark the KPLR000757450-2012121044856 row in the first page and press the 'Plot marked Light curves' button at the top of the list.

- Zoom into the red curve around (1200.6964,11775.290) to obtain a transit representation like Figure 1.

- To visualize the orbital period showed in Figure 2, zoom into the red curve from $(1190.1292,12980.986)$ to $(1202.8074,10357.050)$

For the sonification of the light curves:

- Press the 'Create png Image' button (just below the light curve) and save the file.

- Inside Sonifigrafer user interface, open the '.png' file and press 'Play'.

- Adjust the base and filter's frequencies for sound response optimization.

- The complete configuration, including a copy of the image, can be saved and recalled.

The prototype also includes a 'Planet Hunters TESS' button that links to this online classification project [4]. To sonify its light curves with Sonifigrapher just choose an image, save it as PNG file or make a screenshot, and load it using the synthesizer's 'Open file' button.

\section{TASK-BASED VALIDATION EXAMPLES}

In the second phase of this project, the Planet Hunters TESS light curves [4], [5] have been used with the double intention of testing the possibilities for exoplanet transits auditory detection and validating the prototype with the exploration of a different data set. This open collaborative project provides an interactive light-curve classification tool in which final users can mark observed transits and generate discussion forums.

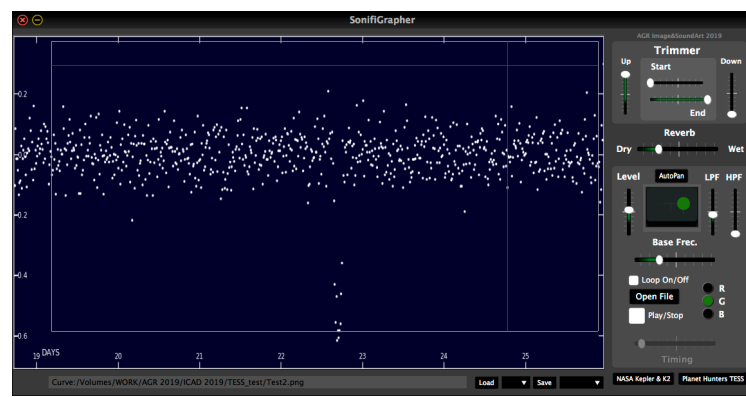

Figure 7: Sonifigrapher interface capture during a Planet Hunters' light curve sonification [4]. Single transit detected.

Intensive testing has been made to evaluate the synthesizer's behavior with this data set. Acting over the base frequency, trimmer controls and filters, auditory transit detection has been satisfactory achieved for the deepest transits, enhancing the graphic information with a real time easily identifiable sonic cue. Several video examples showing the effectiveness of the prototype in these situations are available at:

https://archive.org/details/transits
A more detailed auditory analysis of the curves is also possible acting over the 'start' and 'end' trimmer controls and controlling the playback speed. The smallest amplitude variations in the light curves can be perceived using higher base frequencies and slower velocities. Extremely slow singlepass playback allows bright points discrimination and the creation of chords and electric piano-like arpeggios. This sound sequences can also be repeated, creating eight-seconds loops from the graphic information between markers. Five descriptive light-curve samples are included for exploring the synthesizer. Listening to some examples is also possible in video format, via the Sonifigrapher download page.

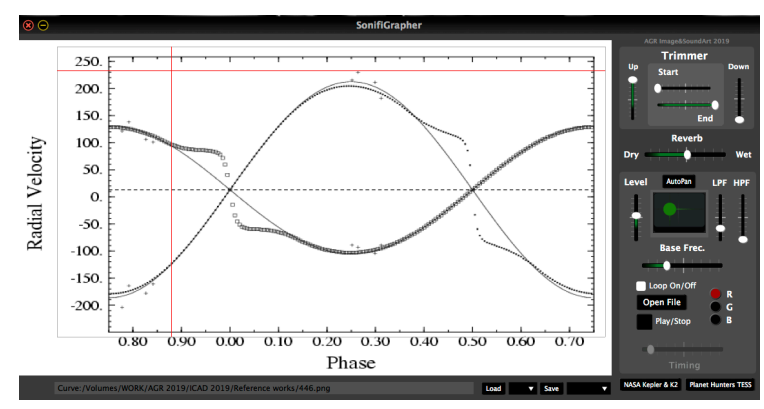

Figure 8: Sonifigrapher interface capture during the sonification of CX Aqr star. Radial velocity vs phase [38].

For final testing and prototype validation, the Catalog and Atlas of Eclipsing Binaries (CALEB) [39] and the CSI 2264 CoRoT's light curves [40] have also been used. A user experience study based in this last catalog is expected to be the third phase of the synthesizer's development project with the intention of providing useful information from both specialized and non-specialized users.

\section{CONCLUSION}

Although CSound is not an image-processing oriented programming language, it allows the development of multimodal interactive software tools that can bring sonification closer not only to scientific specialized audiences but also to students at any level and in any field of knowledge. "Pairing data sonification with data visualization is a synergistic tool for augmenting both visualization and sonification, and can highlight connections or correlations between variables" Scaletti, 2018 [32].

Cabbage's VST exporting options, in conjunction with CSound's never-ending programming and processing possibilities, establish a multidisciplinary natural connection between science-oriented sonifications and music creation environments that opens the possibilities of both disciplines and allows constant feedback between them.

If we look at education and music as relevant parts of our everyday life, it seems important to increment the efforts invested in the development of new multimodal and interdisciplinary sonification tools designed to bring science closer to people. Sonification can represent any kind of information and its unique communication and engagement capabilities could be considered and included when developing institutional education materials to normalize the use of sonifications in the next generation of digital natives. As highlighted by Quinton et al. [41], teaching how to listen seems crucial in the acceptance of sonification as a data analysis tool. 
On the other hand, and according to Scaletti's distinctions between sonification and music [32], the use of sonified data as a sound source for the creation of original music material represents an open field with full potential to build a bridge between the sonification processes and general public. Data-driven virtual instruments' development projects provide the possibility of acting in both public-private, known-unknown and interactive-fixed areas of Scaletti's Sonification Space, generating environments of interest for the sonification community to explore.

\section{REFERENCES}

[1] Vickers, P. (July 2005). Wither and wherefore the Auditory Graph? Abstractions and Aesthetics in Auditory and Sonified Graphs, Proceedings of the $11^{\text {th }}$ International Conference on Auditory Display. (ICAD), Limerick, Ireland.

[2] https://www.nasa.gov/kepler/education/getlightcurves

[3] http://archive.stsci.edu/kepler/data_search/search.php

[4] https://www.zooniverse.org/projects/nora-doteisner/planet-hunters-tess

[5] https://tess.mit.edu/

[6] https://docs.lightkurve.org/

[7] Georgakki, A. May (2005). The grain of Xenakis' Technological thought in the Computer Music research of our days, Proceedings of the International Symposium Iannis Xenakis, Athens, Greece.

[8] Nelson, P. (1997). The UPIC system as an instrument of learning. Organised Sound, 2(1), 35-42.

[9] Xenakis, I. (1992). Formalized Music. Thought and Mathematics in Music. Hillsdale, NY: Pendragon Press.

[10] http://www.centre-iannisxenakis.org/cix_upic_presentation?lang=en

[11] Jacquemin, G., Coduys, T. and Ranc, M. (May 2012). IanniX 0.8. Journées d'Informatique Musicale (JIM), Mons, Belgium.

[12] IanniX software, accessed March 2019: https://www.iannix.org/en/

[13] Walker, B. N. and Cothran, J. T. (July 2003). Proceedings of the 2003 International Conference on Auditory Display, Boston, USA.

[14] Ferguson, J. (2016). Bell3D: An Audio-based Astronomy Education System for Visually-impaired Students. CAPjournal, No.20, pp35.

[15] Diaz Merced, W. L. (2013). Sound for the exploration of space physics data. (Doctoral Thesis). University of Glasgow.

[16] Diaz-Merced, W. L., Candey, R.M., Brickhouse,N., Schneps, M., Mannone, J.C., Brewster, S. and Kolenberg, K. (2012). Sonification of Astronomical Data. New Horizons in Time-Domain Astronomy Proceedings IAU Symposium No. 285, 2011. R.E.M. Griffin, R.J. Hanisch \& R. Seaman, eds.

[17] http://archive.stsci.edu/kepler/condition_flag.html

[18] https://keplergo.arc.nasa.gov/PyKEprimerLCs.shtml

[19] http://archive.stsci.edu/kepler/manuals/archive_manual.pdf

[20] https://www.nasa.gov/feature/jpl/20-intriguing-exoplanets

[21] https://exoplanetarchive.ipac.caltech.edu/

[22] Seager, S. \& Mallén-Ornelas, G. (2002). A Unique Solution of Planet and Star Parameters from an Extrasolar Planet Transit Light Curve. Retrieved from: https://iopscience.iop.org/article/10.1086/346105/fulltext/

[23] Alapini Odunlade, A. E. P. (2010) Transiting exoplanets: characterization in the presence of stellar activity. Doctoral Thesis. University of Exeter.
[24] Winn, N. J. (2010). Transits and Occultations. Retrieved on March 2019 from: https://arxiv.org/abs/1001.2010

[25] CSound software, accessed March 2019: http://www.csounds.com/

[26] CSound and Python API, accessed March 2019: http://floss.booktype.pro/csound/c-python-in-csoundqt/

[27] Cabbage software accessed March 2019: http://cabbageaudio.com/

[28] Steinke, G. April 1966. Experimental Music with the Subharchord. Subharmonic Sound Generator, Journal of the Audio Engineering Society, vol.14, no. 2, pp. 141.

[29] Vercoe, B. MIT Media Lab et al. The Canonical Csound Reference Manual. Retrieved from: http://www.csounds.com/manual/html/

[30] Boulanger, R. (Ed.) (2000). The Csound Book: Perspectives in Software Synthesis, Sound Design, Signal Processing, and Programming. Cambridge, MA, USA: MIT Press.

[31] McCurdy, I. accessed March 2019, http://iainmccurdy.org/

[32] McLean, A. and Dean, T. (Editors) (2018). The Oxford Handbook of Algorithmic Music, Oxford University Press, New York, USA, ch.21.

[33] Herman, T. June. (2008). Taxonomy and definitions for sonification and auditory display. Proceedings of the $14^{\text {th }}$ International Conference on Auditory Display. Paris, France.

[34] Herman, T., Hunt, A. y Neuhoff, J. G. (Eds.) (2011). The Sonification Handbook. Logos Verlag, Berlin, Germany.

[35] De Campo, A. (February 2009). Science by ear. An interdisciplinary Approach to Sonifying Scientific Data. (Thesis). University for Music and Dramatic Arts, Graz.

[36] Vogt, K. (June 2010). Sonification of Simulations in Computational Physics. (Thesis). Institute for Electronic Music and Acoustics, University of Music and Performing Arts, Graz, Austria.

[37] Kramer, G., Walker, B., Bonebright, T., Cook, P., Flowers, J. H., Miner, N. and Neuhoff, J. (2010). Sonification Report: Status of the Field and Research Agenda. Faculty Publications, Department of Psychology. Paper 444. Retrieved from: http://digitalcommons.unl.edu/psychfacpub/444

[38] http://caleb.eastern.edu/model_display.php? model_id=446

[39] http://caleb.eastern.edu/

[40] https://irsa.ipac.caltech.edu/data/SPITZER/CSI2264/lcur ves_corot.html

[41] Quinton, M., McGregor, I. and Benyon, D. (June 2018). Investigating Effective Methods of Designing Sonifications. Proceedings of the $24^{\text {th }}$ International Conference on Auditory Display. (ICAD), Michigan, USA.

\section{ACKNOWLEDGMENT}

This research has made use of the NASA/ IPAC Infrared Science Archive, which is operated by the Jet Propulsion Laboratory, California Institute of Technology, under contract with the National Aeronautics and Space Administration.

Special thanks to Helen Gräwert for reading data driven projections and to Ruth Capó Mesa for listening to the stars. 